\title{
Hyponatriämie wird bei geriatrischen Klinikpatienten unterschätzt
}

— Bis zu 30\% aller hospitalisierten Patienten haben eine Hyponatriämie (Serum-Natrium $<135 \mathrm{mmol} / \mathrm{l}$ ), deren klinische Bedeutung häufig unterschätzt wird. Vor allem bei geriatrischen Patienten mit typischen "Alterssymptomen" wie Gang- und Gedächtnisstörungen, Schwindel, Lethargie oder Verwirrtheit sollte an eine Hyponatriämie als mögliche Ursache gedacht werden.

Eine chronische Hyponatriämie sei auch prognostisch relevant, betonte Prof. Markus Gosch, Geriater am Klinikum Nürnberg. Der Krankenhausaufenthalt der betroffenen
Patienten sei verlängert und die Mortalität erhöht. Zudem sei wegen der Gangstörungen das Sturz- und Frakturrisiko erhöht.

\section{Zur Volumenreduktion bei SIADH hat sich Tolvaptan bewährt}

Bei bis zu einem Drittel der Betroffenen ist die Hyponatriämie Folge eines Syndroms der inadäquaten $\mathrm{ADH}$-Sekretion (SIADH), das durch Infektionen, Asthma, COPD, Karzinome, ZNS-Erkrankungen sowie Medikamente ausgelöst werden kann. Aufgrund der gesteigerten Sekretion des antidiureti- schen Hormons (ADH) ist die Ausscheidung von Wasser über den Urin verringert. Bei diesen Patienten kann zur Volumenreduktion der orale Vasopressin-V2-RezeptorAntagonist Tolvaptan (Samsca ${ }^{\oplus}$ ) eingesetzt werden. Die Behandlung muss im Krankenhaus eingeleitet werden. Die Natriumspiegel sollten dabei engmaschig kontrolliert werden, sagte Gosch. Bei den meisten $\mathrm{Pa}$ tienten stiegen die Serumnatriumwerte rasch an, und auch die klinische Symptomatik bessere sich oft sehr schnell.

Roland Fath

Fachpresseworkshop "Hyponatriämie in der Geriatrie: dem diagnostischen Chamäleon auf der Spur", Nürnberg, 10.10.2017, Veranstalter: Otsuka Pharma

\section{Projekt InTherAKT zur Verbesserung der medikamentösen Versorgung im Alter}

— Vor rund zwei Jahren wurde das Projekt InTherAKT zur Verbesserung der medikamentösen Versorgung in Altenhilfeeinrichtungen gestartet (www.interakt.de). Herzstück des Projekts ist eine Online-Plattform, die an rund 90 Patienten überprüft und optimiert wurde.

Wie der Projektleiter Prof. Jürgen Osterbrink, Salzburg, zum Hintergrund erläuterte, seien betagte und hochbetagte Menschen besonders häufig von unerwünschten Arzneimittelinteraktionen betroffen. Vielfach sei- en die Interaktionen das Resultat nicht abgestimmter Prozesse der Behandler. Ein Ziel des Projektes InTherAKT besteht deshalb darin, die Kommunikation und Kooperation zwischen den Berufsgruppen zu optimieren. Dazu wurden alle Beteiligten nicht nur online, sondern auch in Präsenzveranstaltungen geschult.

\section{Bis zu fünf Medikamente gestrichen}

In InTherAKT wird die Angemessenheit der Medikation anhand des "Medication Appro- priateness Index" (MAl) erhoben. Hier ergab sich eine Verbesserung um durchschnittlich $25 \%$. Besonders deutlich zeigte sich der Erfolg bei Patienten, die vorher sehr viele Medikamente einnehmen mussten. Im Einzelfall konnte die Zahl der Medikamente um bis zu fünf Präparate reduziert werden. Damit unterstreichen die Ergebnisse die Notwendigkeit der interprofessionellen Zusammenarbeit, bei der jede Berufsgruppe ihre Kompetenzen einbringt.

Dr. Matthias Herrmann

Lunch-Symposium „Polypharmazie in der Altenhilfe - Learnings auch für die Schmerztherapie", Deutscher Schmerzkongress, Mannheim, 12.10.2017, Veranstalter: Grünenthal

\section{Vorsicht Sturzgefahr: Kraft- und Gangtraining bei vielen älteren Schwindelpatienten erfolgreich}

\begin{abstract}
Schwindel bei geriatrischen $\mathrm{Pa}$ tienten lässt sich in Vertigo, lokomotorischen Schwindel, präsynkopalen Schwindel und Mischformen unterteilen. Charakteristisch und am häufigsten bei den über 80-Jährigen ist der lokomotorische Schwindel mit Gangstörungen.
\end{abstract}

\section{So gehen Sie therapeutisch vor}

Therapeutische Ansatzpunkte sind zunächst die Suche und Behandlung eventuell vorhandener monokausaler Schwindelursachen, so der Geriater Prof. Karl-Günter Gaßmann vom GeriatrieZentrum Erlangen. Bei multifaktoriellem Schwindel seien das Training von Gangsicherheit, Gleichgewicht, Kraft- sowie ein Verhaltenstraining gegen die Angst vor dem Stürzen indiziert und oft sehr erfolgreich. Nimmt der Patient schwindelinduzierende Medikamente wie Sedativa, zentral wirkende Analgetika, An- tihypertensiva, Spasmolytika oder Parkinsonmedikamente, sollte eine Umstellung oder ein Auslassversuch mit nachfolgendem Ausschleichen probiert werden.

Die medikamentöse Schwindeltherapie greife bei gewissen Indikationen, sollte aber keine Dauertherapie sein. Als einziges natürliches Präparat wird Vertigoheel ${ }^{\circledR}$ bei akutem Schwindel seit einem Jahr in der hausärztlichen DEGAM-Leitlinie empfohlen. Wegen des Fehlens von Neben- und Wechselwirkungen eignet es sich vor allem für multimorbide ältere Patienten.

Dr. Andreas Häckel

Lunchsymposium "Schwindel - eine interdisziplinäre Herausforderung", Kongress der Deutschen Gesellschaft für Geriatrie (DGG), Frankfurt am Main, 29.09.2017, Veranstalter: Heel 